\title{
Genetics Of Chronic Obstructive Pulmonary Diseases: A Mare's Nest
}

\section{Mrinal Ranjan Srivastava ${ }^{1}$, Anu Chandra ${ }^{2}$, Farzana Mahdi $^{2}$, Sharique Ahmad $^{3}$, Sarita Choudhary ${ }^{4 *}$ and Makardhwaj Prasad ${ }^{5}$}

${ }^{1}$ Assistant Professor, Department of Community Medicine, Phulo Jhano Medical College, Dumka, Jharkhand, India

${ }^{2}$ Professor, Department of Biochemistry, Era's Lucknow Medical College and Hospital, Lucknow, Uttar Pradesh, India

${ }^{3}$ Professor, Department of Pathology, Era's Lucknow Medical College and Hospital, Lucknow, Uttar Pradesh, India

${ }^{4}$ Tutor, Department of Anatomy, Saheed Nirmal Mahto Medical College and Hospital, Dhanbad, India

${ }^{5}$ Department of Anatomy, Saheed Nirmal Mahto Medical College and Hospital, Dhanbad, India

*Corresponding Author: Sarita Choudhary, Tutor, Department of Anatomy, Saheed Nirmal Mahto Medical College and Hospital, Dhanbad, India.
Received: March 30, 2021

Published: October 05, 2021

(C) All rights are reserved by Sarita

Choudhary., et al.

\begin{abstract}
Chronic obstructive pulmonary disease (COPD) is a major cause of morbidity and mortality across the globe. According to World Health Organization estimates, 65 million people have moderate to severe COPD. It has been responsible for changing the diseases spectrum spanning continents. Epidemiological data suggest genetics to be one of those factors, as COPD is known to aggregate in families with a stronger correlation between parents and children or siblings than between spouses. Twin and segregation studies provide evidence that genetic predisposition plays a role in COPD and had indicated that the genetic background of COPD is composed of several genes with small effects, rather than a single major gene.
\end{abstract}

Keywords: COPD; ADBR2 gene; MMP; MBL2 Gene; NAC

\section{Introduction}

Chronic obstructive pulmonary disease (COPD) is a major cause of morbidity and mortality across the globe. According to World Health Organization estimates, 65 million people have moderate to severe COPD. More than 3 million people died of COPD in 2005 corresponding to $5 \%$ of all deaths globally and it is estimated to be the third leading cause of death by 2030 [1]. Chronic obstructive pulmonary disease (COPD) is a major health problem as it causes increasing mortality and morbidity in all industrial countries [1,2]. Progressive irreversible airflow limitation is the central pathogenetic aspect in COPD. However, the disease is not only determined by pulmonary function, but by airway tissue inflammation, airway remodeling and systemic consequences. The most important risk factor in the Etiopathogenesis of COPD is cigarette smoking. Nev- ertheless, only $10 \%$ - $20 \%$ of all heavy cigarette smokers develop COPD. There is strong evidence for a relationship between decline in lung function and smoking behavior. However, smoking account only for about $15 \%$ of lung function variability [3]. These observations demonstrate that next to smoking other factors seems to be of importance for the development of the disease. Epidemiological data suggest genetics to be one of those factors, as COPD is known to aggregate in families [4], with a stronger correlation between parents and children or siblings than between spouses [5]. Twin [6] and segregation studies provide evidence that genetic predisposition plays a role in COPD and had indicated that the genetic background of COPD is composed of several genes with small effects, rather than a single major gene [7]. 


\section{Epidemiology and magnitude of COPD in India}

India has $18 \%$ of the global population and an increasing burden of chronic respiratory diseases. India has a disproportionately high burden of chronic respiratory diseases [8].

With the pace of demographic and epidemiological transitions, the changes in socio-behavioral dimensions and their impacts on population health are evident. As on 2016, three out of five leading causes of mortalities constitute non-communicable diseases whereas COPD is the second biggest cause of death in India today. It became fourth leading cause of years of life lost in Empowered Action Group (EAG) States including Bihar, Jharkhand, Madhya Pradesh, Chhattisgarh, Odisha, Rajasthan, Uttar Pradesh and Uttarakhand [9]. Different studies have revealed varying range of prevalence of COPD in different states. The prevalence ranged between 2 to $22 \%$ among the men and 1.2 to $19 \%$ among women in different population-based studies across India [10]. Certain questions still remain to be answered such as what are the factors that lead to region-specific variations in common respiratory diseases in India. The actual practice patterns and socioeconomic impact of these common respiratory ailments need to be known. The impact of seasonal trends in occurrence or exacerbation of these diseases is also not known. Obstructive lung diseases are major contributors to adverse respiratory health status of a country, and control of these diseases is of paramount importance for any health-care system. Finally, looking at the rising burden of respiratory diseases, especially obstructive lung diseases in India, extraordinary efforts need to be undertaken in order to control these problems.

\section{COPD-A paradigm shift}

The extraordinary changes in the last two decades has liberated our strict and centric Forced Expiratory Volume in 1 second (FEV1) view on COPD.As our understanding of this complex condition improves we would indeed see many other factors unfolding. In COPD we need integration of multilevel data (environmental, clinical, biological and genetic). We now have an interesting prospects of identification of genetic markers that facilitate the assessment of risk of future events-lung function decline, co morbidities, exacerbations). a very recent prespecified analysis in 5125 patients with COPD included in the Prevention Of Exacerbations with Tiotropium in COPD (POET-COPD) trial showed that polymorphisms of the $\beta_{2}$-adrenergic receptor (ADRB2) gene (Arg16Gly (rs1042713) and Gln27Glu (rs1042714)) did indeed influence the effect of long-acting bronchodilators (tiotropium vs salmeterol) in the prevention of exacerbations [11].

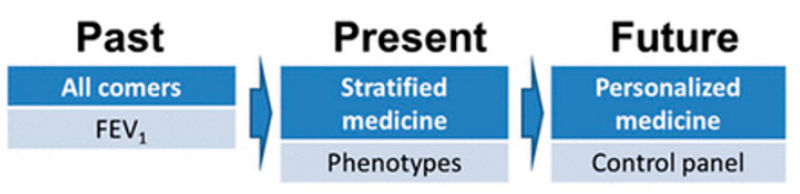

Figure 1

Most of the studies of genetic polymorphisms and COPD have been done in western countries and Chinese population. There is a serious dearth of information of genetic associations and the behavior of COPD types based on these polymorphisms in Indian population. We propose to test polymorphisms of ADRB2 gene and mEPHX1 gene, MBL2 gene, MMP-2/TIMP-2 and the impact of treatment, patient related outcome in phenotypes with expressions of these polymorphisms.

It is well known that chronic obstructive pulmonary disease (COPD) is strongly associated with genetic factors and that susceptibility to COPD depends in part on the genetic phenotypes and gene polymorphism of a variety of factors involved in the pathogenesis of COPD, such as inflammatory cytokines, proteases, antiproteases, oxidoreductases, and detoxifying enzymes. There is good evidence to suggest that increasing oxidative stress is a key factor in the pathogenesis of COPD [12-14]. The body has a perfect enzymatic and nonenzymatic antioxidation system to cope with oxidative stress and protect the body from attack by oxidants. The main known oxidation inhibition enzymes in the body including glutathione-S-transferase, microsomal epoxide hydrolase (EPHX1), and heme oxygenase, hydrolyze and inactivate oxygen metabolites, thus fighting against or neutralizing the oxidative damage caused by oxidative stress, and eventually maintaining the dynamic balance of oxidation/antioxidation in the body. When the production of oxidation inhibitors is decreased or their activity is diminished as a result of genetic variation, the dynamic balance of oxidation/ antioxidation is lost, leading to oxidative damage.

Although smoking is the major causal factor in the development of chronic obstructive pulmonary disease (COPD), only 10-20\% of chronic heavy cigarette smokers develop symptomatic COPD, which suggests the presence of genetic susceptibility. The human microsomal epoxide hydrolase (EH) is a metabolizing enzyme which involves the process of numerous reactive epoxide interme- 
diates and contains polymorphic alleles which are associated with altered EH activity and may be linked to increased risk for COPD.

Chronic obstructive pulmonary diseases and EPHX-1 gene and polymorphisms

Park JY., et al. in a study To determine whether the $E H$ polymorphisms contributed to increased risk for COPD, prevalence of the EH codons 113 and 139 polymorphisms were compared between COPD patients and controls using a PCR-RFLP analysis using genomic DNA isolated from 131 COPD patients and 262 individually matched controls by age ( \pm 5 years) among Caucasians with 1:2 ratio. Significantly increased risk for COPD was observed for subjects with the $\mathrm{EH}^{113 \mathrm{His} / \mathrm{His}}$ genotypes $(\mathrm{OR}=2.4,95 \% \mathrm{CI}=1.1-5.1)$. These results were consistent with the fact that a significant trend towards increased risk was observed with predicted less protective EH codon 113 genotypes ( $p=0.03$, trend test). A similar association was not observed for EH codon139 polymorphism [15].

Zidzik J., et al. in contrast in another study to determine the risk of chronic obstructive pulmonary disease (COPD) associated with polymorphisms in the glutathione S-transferase (GST) M1, GST T1, and microsomal epoxide hydrolase (EPHX1) genes in a cohort of Slovak population observed that Although none of the tested gene polymorphisms was significantly related to an increased risk of COPD alone, the results suggested that the homozygous exon $3 \mathrm{mu}-$ tant variant of EPHX1 gene in the combination with GSTM1 null genotype is a significant predictor of increased susceptibility to COPD in the Slovak population. The findings of the present study emphasize the importance of detoxifying and antioxidant pathways in the pathogenesis of COPD [16].

Cheng Sl., et al. the genotypes of 184 patients with COPD and 212 control subjects were determined by polymerase chain reaction followed by restriction fragment length polymorphism analysis of the mEPHX, GSTM1, GSTT1 and GSTP1 genes. All subjects were smokers or exsmokers. The proportion of GSTM1-null genotypes was significantly higher in patients with COPD than in control subjects (61.4 versus $42.5 \%$ ). No differences were observed in the frequency of polymorphic genotypes for mEPHX, GSTT1 and GSTP1. During combined analysis of genetic polymorphisms for mEPHX, GSTM1 and GSTP1, it was found that there are strong indicators for susceptibility to COPD (genotype combination with at least one mutant mEPHX exon-3 allele (histidine 113), GSTM1 null and ho- mozygous for the GSTPI isoleucine 105 allele). The frequencies of homozygous mutant alleles of mEPHX exon 3 and the GSTMI-null genotype were significantly higher in patients with severe COPD (forced expiratory volume in one second of $<35 \%$ of the predicted value). It is proposed that the combination of genetic variants including at least one mutant microsomal epoxide hydrolase exon3 allele and glutathione S-transferase M1-null and homozygous isoleucine 105 glutathione S-transferase P1 genotypes are significant indicators of susceptibility to chronic obstructive pulmonary disease in the Taiwanese population. In addition, the homozygous variant of microsomal epoxide hydrolase exon 3 and the glutathione S-transferase M1-null genotype are independent risk factors for developing severe chronic obstructive pulmonary disease [17].

Matheson Mc., et al. in contrast investigated the influence of single nucleotide polymorphisms (SNPs) in EPHX1 on well-characterized COPD and intermediate phenotypes. A total of 1,232 participants completed a detailed respiratory questionnaire and spirometry. From this sample, 72 COPD cases (FEV1/FVC $<0.70$ and FEV1 $<80 \%$ predicted) and 220 control subjects (no respiratory symptoms and normal lung function) were selected for analysis. The EPHX1 exon 3 and EPHX1 exon 4 polymorphisms were carefully genotyped to avoid error using several methods. They found that the EPHX1 exon 3 polymorphism was not associated with an increased risk of COPD, nor was the EPHX1 exon 4 polymorphism [18].

\section{Nacetylcysteine and association with EPHX-1 gene}

Numerous studies have been performed over the last two decades to assess whether NAC has potential therapeutic effects in patients with COPD. No previous studies have shown a significant increase in FEV1 with either high-dose or low-dose NAC, although some studies reported improvements in some parameters of lung function after treatment with NAC. For example, Stav and Raz found that inspiratory capacity and FVC were higher, in particular, post exercise, after treatment with NAC $600 \mathrm{mg}$ twice daily compared with placebo [4], and Tse., et al. found that high-dose NAC resulted in significantly improved small airway function [5]. Some of the interindividual differences in effect associated with NAC treatment might be explained by genetic polymorphism of the antioxidase.

Zuin., et al. to investigate the efficacy and tolerability of highdose $\mathrm{N}$-acetylcysteine (NAC) in the treatment of patients with ex- 
acerbations of chronic obstructive pulmonary disease (COPD). The primary objective was to assess the proportion of patients with normalised C-reactive protein (CRP) levels. They also assessed the effects on interleukin (IL)-8 levels, lung function and symptoms. They concluded that treatment with NAC $1200 \mathrm{mg} /$ day improved biological markers and clinical outcomes in patients with COPD exacerbations. It is speculated that the effect of NAC on inflammatory markers may be due to both mucolytic and antioxidant properties [19].

Shen Y., et al. in a systematic review and metanalysis showed that high-dose NAC can reduce both the total number of exacerbations ( $\mathrm{RR}=0.59,0.47$ to $0.74,95 \% \mathrm{CI}, \mathrm{p}<0.001$ ) and the proportion of patients with at least one exacerbation $(R R=0.76,0.59$ to 0.98 , $95 \% \mathrm{CI}, \mathrm{p}=0.03$ ). In the low-dose group, subgroup with jadad $\leq 3$ showed a significant decrease (RR $=0.69,0.61$ to $0.77,95 \% \mathrm{CI}, \mathrm{p}<$ 0.001 ) in the proportion of patients with exacerbation, the other subgroup with Jadad score $>3$ showed no significant decrease (RR $=0.98,0.90$ to $1.06,95 \% \mathrm{CI}, \mathrm{p}=0.59$ ). And low-dose NAC showed no benefit in the total number of exacerbations ( $R R=0.97,0.68$ to $1.37,95 \% \mathrm{CI}, \mathrm{p}=0.85$ ). Neither high nor low-dose NAC treatment showed benefit in forced expiratory volume in one second (FEV1) (WMD $=1.08,-9.97$ to $12.13,95 \% \mathrm{CI}, \mathrm{p}=0.85$ ) [20].

Role of Beta2-adrenergic receptor in chronic obstructive pulmonary diseases

COPD is characterized by a persistent airflow limitation that is not fully reversible; thus, the reversibility of airflow limitations in response to a bronchodilator is an important component of COPD. A major cause for COPD is airflow obstruction in the lung and respiratory parenchyma maintained by airway smooth muscle cells. Chronic obstructive abnormality followed by airway remodeling increases the thickness of the airway and causes airflow obstruction [12]. Therefore, genes involved in the regulation of airway smooth muscle tone are good candidates for the genetic predisposition to COPD. Previous studies have found that several genetic variants of ADRB2 are associated with COPD [13], but the real function of these variants is not elaborated, especially the noncoding variants, which might play a more important role in altering ADRB2 expression and further resulting in COPD. So, it is essential and necessary to use new data and multilevel surveys, including genetic association study and functional analysis, to further elucidate the role of ADRB2 noncoding variant in COPD, comprehensively.
Matheson MC., et al. in a study was to examine the associations between the beta (2) AR polymorphisms and risks of asthma, chronic obstructive pulmonary disease (COPD) and respiratory symptoms in a sample of adults. Participants were part of a crosssectional population-based study of risk factors for respiratory disease. A total of 1,090 Caucasian participants completed a detailed respiratory questionnaire, spirometry, methacholine challenge and measurement of gas transfer. Genotyping for beta (2) AR polymorphisms at positions 16 and 27 was performed using the tetra-primer amplification refractory mutation system-polymerase chain reaction (ARMS-PCR) method. Haplotype frequencies for the two polymorphisms were estimated using the E-M algorithm. We found the Arg16 homozygotes had an increased risk of COPD (OR 5.13; 95\% CI 1.40,18.8), asthma (2.44; 1.12,5.31) and symptoms of wheeze $(1.84 ; 1.02,3.35)$. The Gln 27 homozygotes had an increased risk of asthma $(2.08 ; 1.05,4.13)$ and bronchial hyperreactivity (BHR) (1.92; 1.07,3.46). The Arg16/Gln27 haplotype was associated with asthma $(1.63 ; 1.12,2.38)$ and COPD $(2.91 ; 1.42,5.94)$. The Arg16/Gln27 beta(2)AR haplotype is important in COPD, asthma and BHR, and may be associated with more severe respiratory symptoms in middle-aged and older adults [21].

Thomson M., et al. in a study tested the hypothesis that three functional polymorphisms in the ADRB2 gene (Thr164Ile, Gly16Arg and Gln27Glu) are associated with reduced lung function, asthma or chronic obstructive pulmonary disease (COPD). They genotyped 8,971 individuals from the Copenhagen City Heart Study for all three polymorphisms. To validate their findings, they genotyped an additional 53,777 individuals from the Copenhagen General Population Study for the Thr164Ile polymorphism. They identified 60,910 Thr164Ile noncarriers, 1,822 heterozygotes and 16 homozygotes. In the Copenhagen City Heart Study, the Thr164Ile genotype was associated with reduced forced expiratory volume in $1 \mathrm{~s}$ $(\mathrm{FEV}(1)) \%$ predicted (trend $\mathrm{p}=0.01)$ and FEV(1)/forced vital capacity (FVC) ( $p=0.001)$ : Thr164Ile heterozygotes had $3 \%$ and $2 \%$ reduced $\mathrm{FEV}(1) \%$ pred and $\mathrm{FEV}(1) / \mathrm{FVC}$, respectively, compared with noncarriers. The odds ratio for COPD in Thr164Ile heterozygotes was 1.46 (95\% CI 1.05-2.02). In the Copenhagen General Population Study, the Thr164 genotype associated with reduced FEV(1)\% pred ( $\mathrm{p}=0.04$ ) and FEV(1)/FVC ( $<$ 0.001): Thr164Ile homozygotes and heterozygotes had $7 \%$ and $1 \%$ reduced $\mathrm{FEV}(1) \%$ pred and $6 \%$ and $1 \%$ reduced FEV(1)/FVC, respectively, compared 
with noncarriers. The odds ratios for COPD in Thr164Ile homozygotes and heterozygotes were 4.53 (95\% CI 1.54-13.3) and 1.07 (95\% CI 0.92-1.25), respectively. They that ADRB2 concluded that Thr164Ile is associated with reduced lung function and increased risk of COPD in the general population [22].

Vacca G., et al. in a study investigated 190 COPD patients and 172 healthy volunteers in a case-control study. DNA was isolated from whole blood and B2-AR gene polymorphisms Arg/Gly16 and Gln/Glu27 were determined using allele-specific polymerase chain reaction (PCR). It was observed that In COPD patients with Gly/ Gly16 was more frequently than in healthy smokers $(29.47 \%$ COPD versus $18.18 \%$ controls, $p=0.026$ ). All other gene polymorphisms of the ADRB2 gene at codon 16 were equally distributed between groups. ß2-adrenoreceptor gene polymorphisms were neither a cofounder for COPD exacerbations $(\geq 3$ hospitalizations within the last 3 years) nor for disease severity (FEV1 $\leq 30 \%$ predicted). Their study suggests that the Gly16 allele of the ß2-AR gene predisposes to COPD development but not for exacerbation rates and disease severity. In contrast, Gln/Glu27 polymorphism was irrelevant in our COPD cohort [23].

Niu LM., et al. in contrast in a metanalysis of Twelve case-control studies and eight cross-sectional studies analysed that Compared to the control ( $\mathrm{n}=1225)$, neither Gly/Gly ( $\mathrm{n}=527)$ nor Arg/Arg ( $\mathrm{n}$ = 422) homozygotes at position 16 demonstrated increased susceptibility to COPD, with odds ratios (ORs) of 0.95 (95\%CI (0.68, 1.31), $\mathrm{z}=0.33, \mathrm{P}=0.740)$ and $0.82(95 \% \mathrm{CI}(0.52,1.28), \mathrm{z}=0.88$, $\mathrm{P}=0.381$ ), respectively. Similar results were obtained for position 27 , with ORs of 0.97 (95\%CI $(0.77,1.23), z=0.21, P=0.833$ ) for Glu/Glu homozygotes $(\mathrm{n}=357)$ and $0.82(95 \% \mathrm{CI}(0.53,1.29), \mathrm{z}$ $=0.85, \mathrm{P}=0.393$ ) for $\mathrm{Gln} / \mathrm{Gln}$ homozygotes $(\mathrm{n}=704)$ (control = 1183). In patients with COPD, Arg/Arg homozygotes $(n=41)$ had a similar FEV1\% compared with Gly/Gly homozygotes ( $\mathrm{n}=102$ ) (standardized mean difference $(\mathrm{SMD})=0.88,95 \% \mathrm{CI}(-0.85,2.62), \mathrm{z}$ $=1.00, \mathrm{P}=0.319)$. The genotype distribution was different between Caucasian and Asian populations (all $\mathrm{P}<0.05$ except the genotype Arg/Gly) for both position 16 and 27.Polymorphisms of ADRB2 at positions 16 and 27 did not change the risk of COPD nor affect lung function or disease severity. The genotype distribution for these polymorphisms was different between Caucasian and Asian populations [24].
Beta2-adrenergic receptor genetic polymorphisms and bronchodilator responses in patients with COPD

Hizawa N., et al. stated that Several studies have established that two common nonsynonymous polymorphisms in the beta2-adrenergic receptor gene (ADRB2), Arg16Gly and Gln27Glu, have important effects in modulating responses to beta2-agonists; however, the effects of these polymorphisms on responses to beta2-agonists in patients with COPD is unknown. A total of 246 patients with COPD who were participants in a longitudinal study of COPD (ie, the Hokkaido COPD cohort study) were studied. They compared short-term bronchodilator responses (BDRs) to salbutamol according to ADRB2 genotypes at codons 16 and 27.

The presence of the Arg16 allele was associated with lower BDRs to beta2-agonist inhalation. The mean (+/-SD) log (postbronchodilator FEV1-prebronchodilator FEV1) values of Gly16 homozygotes ( $n=65)$, Arg16Gly16 heterozygotes $(n=106)$, and Arg16 homozygotes $(\mathrm{n}=75)$ were $2.19+/-0.43,2.09+/-0.42$, and $2.01+/-0.42$, respectively $(\mathrm{p}<0.05)$. The genetic effects of the Arg$16 \mathrm{Gly}$ polymorphism were independent of the severity of airflow limitation, age, and smoking status. The most common Arg16Gln27 haplotype was also significantly associated with decreased BDRs to salbutamol $(\mathrm{p}<0.01)$.The genetic effects of ADRB2 gene polymorphisms may explain some of the variability in response to therapeutic doses of a short-acting beta2-agonists in patients with COPD [25].

\section{Mannose binding lectins (MBL) and COPD association}

Yang IA., et al. in a study measured Serum MBL as mannan-binding activity in a subgroup of 82 stable COPD patients. Frequency of COPD admissions for infective exacerbation was ascertained for a 2-year period. The MBL2 codon 54 B allele reduced serum MBL in COPD patients. In keeping, patients carrying the low MBLproducing $B$ allele had increased risk of admission for infective exacerbation (OR 4.9, P(corrected) $=0.011)$. No association of MBL2 genotype with susceptibility to COPD was detected. In COPD, serum MBL is regulated by polymorphism at codon 54 in its encoding gene. Low MBL-producing genotypes were associated with more frequent admissions to hospital with respiratory infection, suggesting that the MBL2 gene is disease-modifying in COPD. MBL2 genotype should be explored prospectively as a prognostic marker for infection risk in COPD [26]. 
Rantala A., et al. in an exploratory study investigated the association of serum MBL concentrations and MBL2 gene polymorphisms with respiratory tract infections in young men. They genotyped 6 single-nucleotide polymorphisms (SNPs) in the promoter region (alleles $\mathrm{H} / \mathrm{L}, \mathrm{X} / \mathrm{Y}$, and $\mathrm{P} / \mathrm{Q}$ ) and exon 1 (variant alleles $\mathrm{B}, \mathrm{C}$, and $\mathrm{D}$ and wild-type allele $\mathrm{A}$ ) of the MBL2 gene by real-time polymerase chain reaction and measured serum MBL concentrations in 111 Finnish military recruits with asthma and 362 without asthma. An MBL level below the median concentration was a significant risk factor for infections (asthma status-adjusted odds ratio [OR], 2.5 [95\% confidence interval $\{\mathrm{CI}\}, 1.4-4.5]$ ). Among the 6 SNPs, there was a significant association between the promoter $\mathrm{Y} / \mathrm{Y}$ genotype and infections (OR, 2.3 [95\% CI, 1.2-4.4]) and a borderline significant association between exon 1 variant alleles and infections (OR, 1.7 [95\% CI, 0.9-3.1]), after adjustment for asthma status These preliminary results suggest, for the first time, an association between MBL level and respiratory tract infections in young men and a possible association between infections and MBL2 polymorphisms [27].

Eagan TM., et al. in contrast 415 COPD patients and 231 healthy subjects, aged 40-75, were examined in 2006/07. Plasma MBL levels were determined by ELISA. Low or deficient MBL levels were defined as plasma levels below $500 \mathrm{ng} / \mathrm{mL}$ or $100 \mathrm{ng} / \mathrm{mL}$ respectively. Logistic regression models determined factors associated with MBL deficiency; with explanatory variables study category, sex, age, smoking, comorbid heart disease, and CRP. For COPD severity, explanatory variables were FEV1, exacerbation history, hypoxia, respiratory symptoms, inhaled steroids, and CRP. 18.2\% of healthy subjects and $22.2 \%$ of patients had MBL levels below $100 \mathrm{ng} / \mathrm{mL}$ (p $=0.23$ ); $42.9 \%$ of healthy subjects and $49.6 \%$ of patients had levels below 500ng/mL ( $\mathrm{p}=0.10)$. After adjustment for co-variables, patients had an OR $(95 \% \mathrm{CI})$ of $1.26(0.7,2.2)$ for having MBL levels below $100 \mathrm{ng} / \mathrm{mL}$ compared with healthy subjects, and an OR (95\% CI) of $1.06(0.7,1.7)$ for having levels below $500 \mathrm{ng} / \mathrm{mL}$. Among the COPD patients, none of the disease variables were associated with MBL deficiency. No association was found between MBL deficiency and COPD or COPD severity [28].

\section{Matrix metalloproteinases in COPD}

There is considerable evidence that matrix metalloproteinases (MMPs) are up- and/or downregulated in chronic obstructive pulmonary disease (COPD), particularly in emphysema, in which they probably participate in proteolytic attack on the alveolar wall matrix. Recent data suggest that MMPs also have major roles in driving inflammation or shutting it down, as well as modifying the release of fibrogenic growth factors, processes that are important in the genesis of the various lesions of COPD. In cigarette smokeinduced animal models of emphysema, MMP-12 appears to play a consistent and important role, whereas the data for other MMPs are difficult to interpret. In human lungs, evidence for a role for MMPs is more tenuous and there are numerous contradictions in the literature. Little is known about the effects of MMPs in small airway remodelling, smoke-induced pulmonary hypertension and chronic bronchitis, but MMP-12 participates in experimental small airway modelling. To date, the accumulated data suggest that selective inhibition of MMP-12 might be a viable therapy for emphysema and small airway remodelling, but subtle differences in the functions of MMP-12 in animals and humans mandate caution with this approach. Whether inhibition of other MMPs might be useful is unclear.

Hunninghake MG., et al. stated that Genetic variants influencing lung function in children and adults may ultimately lead to the development of chronic obstructive pulmonary disease (COPD), particularly in high-risk groups. They tested for an association between single-nucleotide polymorphisms (SNPs) in the gene encoding matrix metalloproteinase 12 (MMP12) and a measure of lung function (prebronchodilator forced expiratory volume in 1 second [FEV1]) in more than 8300 subjects in seven cohorts that included children and adults. Within the Normative Aging Study (NAS), a cohort of initially healthy adult men, were tested for an association between SNPs that were associated with FEV1 and the time to the onset of COPD. They then examined the relationship between MMP12 SNPs and COPD in two cohorts of adults with COPD or at risk for COPD. The minor allele (G) of a functional variant in the promoter region of MMP12 (rs2276109 [-82A $\rightarrow \mathrm{G}])$ was positively associated with FEV1 in a combined analysis of children with asthma and adult former and current smokers in all cohorts $(\mathrm{P}=2 \times 10-6)$. This allele was also associated with a reduced risk of the onset of COPD in the NAS cohort (hazard ratio, 0.65 ; $95 \%$ confidence interval [CI], 0.46 to $0.92 ; \mathrm{P}=0.02$ ) and with a reduced risk of COPD in a cohort of smokers (odds ratio, $0.63 ; 95 \% \mathrm{CI}, 0.45$ to $0.88 ; \mathrm{P}=0.005$ ) and among participants in a family-based study of early-onset COPD (P = 0.006).The minor allele of a SNP in MMP12 (rs2276109) is associated with a positive effect on lung function in 
children with asthma and in adults who smoke. This allele is also associated with a reduced risk of COPD in adult smokers [29].

\section{Implications}

Chronic progressive disease like COPD reduces the lifespan and quality of life among affected populations. There is growing evidence for genetic risk factors for COPD: early familial aggregation and linkage analysis studies strongly suggested genetic contributions to COPD, and recent genome-wide association studies have identified several genomic regions that are clearly related to COPD susceptibility. However, despite recent advances in COPD genetics, much of the heritability of COPD remains unexplained, and functional studies are only beginning to elucidate a role for the genetic associations that have been identified. Despite this, the future is bright for understanding the genetics of COPD. Improvements in COPD phenotyping, collaborations among COPD study cohorts, and novel integrative approaches to identifying genetic markers all promise to unravel much of this missing heritability and ultimately lead to improvements in our understanding of COPD susceptibility and treatment.

\section{Conclusion}

Blaming the patients for their previous exposure to risk factors and treating them with palliative care is not a cost effective strategy in India. Further research would definitely help to answer some complex question such as efficacy of drugs related to the genetic polymorphisms in Indian population and gene polymorphisms associated predication of exacerbations in COPD. Understanding of Dynamics of COPD would definitely in the longer run help in containing the disease to a much greater extent.

\section{Bibliography}

1. Pauwels RA., et al. "Global strategy for the diagnosis, management, and prevention of chronic obstructive pulmonary disease. NHLBI/WHO Global Initiative for Chronic Obstructive Lung Disease (GOLD) Workshop summary". American Journal of Respiratory and Critical Care Medicine 163 (2001): 12561276.

2. Hurd S. "Global impact of COPD”. Experimental Lung Research 31 (2005): 57-62.

3. Beck GJ., et al. "Smoking and lung function". American Review of Respiratory Disease Returns 123 (1981): 149-155.
4. Lebowitz MD., et al. "Family aggregation of pulmonary function measurements". American Review of Respiratory Disease Returns 129 (1984): 8-11.

5. Kauffmann F., et al. "Familial factors related to lung function in children aged 6-10 years. Results from the PAARC epidemiologic study". American Journal of Epidemiology 129 (1989): 1289-1299.

6. Redline S. "Genetic and perinatal risk factors for the development of chronic obstructive pulmonary disease". New York: Marcel Dekker (1990).

7. Givelber RJ., et al. "Segregation analysis of pulmonary function among families in the Framingham Study". American Journal of Respiratory and Critical Care Medicine 157 (1998): 14451451.

8. Salvi Sundeep., et al. "The burden of chronic respiratory diseases and their heterogeneity across the states of India: the Global Burden of Disease Study 1990-2016". The Lancet Global Health 6.12 (2018): e1363-e1374.

9. ICMR-PHFI-IHME. India: Health of the Nation's States (2017).

10. Jindal SK., et al. "A review of population studies from India to estimate national burden of chronic obstructive pulmonary disease and its association with smoking". Indian Journal of Chest Diseases and Allied Sciences 43.3 (2001): 139-147.

11. Rabe KF., et al. "Effect of ADRB2 polymorphisms on the efficacy of salmeterol and tiotropium in preventing COPD exacerbations: a prespecified substudy of the POET-COPD trial". Lancet Respiratory Medicine 2 (2014): 44-53.

12. MacNee W. "Pulmonary and systemic oxidant/antioxidant imbalance in chronic obstructive pulmonary disease". Proceedings of the. American Thoracic Society 2.1 (2005): 50-60.

13. Spruit MA., et al. "An official American Thoracic Society/European Respiratory Society statement: key concepts and advances in pulmonary rehabilitation". American Journal of Respiratory and Critical Care Medicine 188.8 (2013): e13-e64.

14. Vestbo J., et al. "Global Strategy for the Diagnosis, Management, and Prevention of Chronic Obstructive Pulmonary Disease: GOLD executive summary". American Journal of Respiratory and Critical Care Medicine 187.4 (2013): 347-365. 
15. Park JY., et al. "Polymorphisms for microsomal epoxide hydrolase and genetic susceptibility to COPD". International Journal of Molecular Medicine 15.3 (2005): 443-448.

16. Zidzik J., et al. "Glutathione S-transferase and microsomal epoxide hydrolase gene polymorphisms and risk of chronic obstructive pulmonary disease in Slovak population". Croat Medical Journal 49.2 (2008): 182-191.

17. Cheng SL., et al. "Genetic polymorphism of epoxide hydrolase and glutathione S-transferase in COPD”. European Respiratory Journal 23 (2004): 818-824.

18. Matheson MC., et al. "Microsomal epoxide hydrolase is not associated with COPD in a community-based sample". Human Biology 78 (2006): 705-717.

19. Zuin R., et al. "High-dose N-acetylcysteine in patients with exacerbations of chronic obstructive pulmonary disease". Clinical Drug Investigation 25.6 (2005): 401-408.

20. Shen Y., et al. "Effect of high/low dose N-acetylcysteine on chronic obstructive pulmonary disease: a systematic review and meta-analysis". COPD 11.3 (2014): 351-358.

21. Matheson MC., et al. "Beta2-adrenergic receptor polymorphisms are associated with asthma and COPD in adults". Journal of Human Genetics 51 (2006): 943-951.

22. Thomsen M., et al. European Respiratory Journal 39.3 (2012): 558-566.

23. Vacca G., et al. "Polymorphisms of the $ß 2$ adrenoreceptor gene in chronic obstructive pulmonary disease". Therapeutic Advances in Respiratory Disease (2009): 3-10.

24. Niu LM., et al. "Effect of polymorphisms in the $\beta 2$-adrenergic receptor on the susceptibility and pulmonary function of patients with chronic obstructive pulmonary disease: a meta analysis". Chinese Medical Journal 125 (2012): 2213-2218.

25. Hizawa N., et al. "Beta2-adrenergic receptor genetic polymorphisms and short-term bronchodilator responses in patients with COPD". Chest 132.5 (2007): 1485-1492.

26. Yang IA., et al. "Mannose-binding lectin gene polymorphism predicts hospital admissions for COPD infections". Genes Immunity 4 (2003): 269-274.
27. Rantala A., et al. "Mannose-binding lectin concentrations, MBL2 polymorphisms, and susceptibility to respiratory tract infections in young men". The Journal of Infectious Diseases 198 (2008): 1247-1253.

28. Eagan TM., et al. "Systemic mannose-binding lectin is not associated with chronic obstructive pulmonary disease". Respiratory Medicine 104 (2010): 283-290.

29. Hunninghake MG., et al. "MMP12, Lung Function, and COPD in High-Risk Populations". The New England Journal of Medicine 361 (2009): 2599-2608.

\section{Volume 5 Issue 10 October 2021}

(C) All rights are reserved by Sarita Choudhary., et al. 